\title{
Assessment of Myocardial CZT SPECT Recording in a Forward-Leaning Bikerlike Position
}

\author{
Mathieu Perrin ${ }^{1}$, Véronique Roch ${ }^{1}$, Marine Claudin ${ }^{1}$, Antoine Verger ${ }^{1-3}$, Henri Boutley ${ }^{4}$, Gilles Karcher ${ }^{1,2,4}$, \\ Cédric Baumann ${ }^{5}$, Nicolas Veran ${ }^{1}$, Pierre-Yves Marie ${ }^{1,2,6}$, and Laetitia Imbert ${ }^{1-3}$ \\ ${ }^{1}$ Department of Nuclear Medicine, CHRU-Nancy, Nancy, France; ${ }^{2}$ Nancyclotep Molecular Imaging Platform, CHRU-Nancy, Nancy, \\ France; ${ }^{3}$ Université de Lorraine, INSERM, UMR 1254, Nancy, France; ${ }^{4}$ Laboratoire de Biophysique and Nancyclotep Molecular \\ Imaging Platform, Université de Lorraine, Nancy, France; ${ }^{5}$ Clinical Research Support Platform, Université de Lorraine, \\ CHRU-Nancy, Nancy, France; and ${ }^{6}$ Université de Lorraine, INSERM, UMR 1116, Nancy, France
}

This prospective randomized study assessed myocardial perfusion imaging with the high-sensitivity D.SPECT cadmium-zinc-telluride camera in a forward-leaning bikerlike position, which may potentially lower diaphragmatic attenuation and reduce breathing-related cardiac motion, in a manner comparable to the prone position proposed with other SPECT cameras. Methods: Patients referred for a stress-rest $99 \mathrm{mTC}$-sestamibi protocol and positioned in the biker position, with the chest leaning forward on the D.SPECT camera-head at $35^{\circ}$ from vertical, had an additional resting D.SPECT recording in the supine position $(n=40)$ or in the sitting position with the back rearward at $30^{\circ}$ from vertical $(n=40)$. Segments with attenuation artifacts were defined as those with less than $65 \%$ uptake but with strictly normal contractility at gated SPECT and no defect reversibility from stress images. Results: The biker position was associated with lower heart-to-detector distances than the supine or sitting positions (both $P<0.001$ ); lower cardiac motion amplitudes, assessed on panograms, than the supine position $(P<$ 0.001 ); and fewer segments with attenuation artifacts than the supine position (on average, $1.10 \pm 1.01$ vs. $1.90 \pm 1.74, P=0.010$ ) or the sitting position $(0.75 \pm 0.93$ vs. $1.38 \pm 1.60, P=0.011)$. Conclusion: Myocardial perfusion images from D.SPECT are enhanced for patients positioned in a forward-leaning bikerlike position comparatively to sitting or supine positions, with a notably lower rate of attenuation artifacts.

Key Words: myocardial perfusion imaging (MPI); CZT camera; left ventricular function; attenuation artifacts; patient position

J Nucl Med 2019; 60:824-829

DOI: 10.2967/jnumed.118.217695

\section{$\mathbf{M}$}

yocardial perfusion SPECT imaging is commonly performed with patients in the supine position and the left or both arms above the head. Unfortunately, the resulting images may be hampered by a diaphragmatic attenuation of the inferior wall, constituting a source of false-positive results, even with the more recent cardiac cadmium-zinc-telluride (CZT) cameras (1-5).

Received Jul. 16, 2018; revision accepted Oct. 15, 2018.

For correspondence or reprints contact: Laetitia Imbert, Médecine Nucléaire, Hôpital de Brabois, CHU-Nancy, Allée du Morvan, 54500 Vandæuvre-les-Nancy, France.

E-mail: I.imbert@chru-nancy.fr

Published online Nov. 2, 2018.

COPYRIGHT (C) 2019 by the Society of Nuclear Medicine and Molecular Imaging.
These attenuation artifacts may be recognized through a particular pattern involving fixed perfusion defects but, unlike myocardial infarction, without any contractility abnormality at gated SPECT $(6,7)$. However, this pattern does not allow the identification of all attenuation artifacts (7), with the need that patient position, and thus attenuation artifacts, should be exactly the same on rest and stress SPECT.

CT-based techniques of attenuation correction are increasingly used in this setting $(7,8)$. They are, however, associated with an increase in patient irradiation, as well as with possible additional artifacts due to the inherent difficulty in matching the SPECT and CT images obtained under very different conditions (8).

Attempts to eliminate artifacts by imaging in the prone position have also been extensively reported (2,5,9-13), with an efficiency not far from that obtained with the CT-based method for preventing inferior attenuation artifacts (5) and with the additional advantage of limiting certain breathing-related artifacts (cranial cardiac drift (12)). However, this method has the disadvantage of being somewhat uncomfortable and has been reported to produce anterior attenuation artifacts $(11,13)$. As a result, imaging protocols mixing SPECT recordings in different positions have been developed and proven relatively efficient $(9,11,14)$, although the latter may be difficult to associate with the somewhat long recording times of low-dose protocols.

Prone positioning may not be proposed to patients when recording with the D.SPECT camera (Spectrum Dynamics) because of the particular geometry of this camera. It may, however, be replaced by a forward-leaning position, similarly to the biker position on a motorbike and previously shown to provide a high diagnostic accuracy in low-dose stress-first protocols (15). We hypothesized that this bikerlike position may potentially lower diaphragmatic attenuation as well as the amplitude of breathing-related cardiac motion, similarly to that documented for the prone position. However, no direct comparison has been conducted to date with the supine or sitting positions, which are currently proposed for the D.SPECT camera. The present prospective randomized study therefore aimed to assess myocardial perfusion imaging recorded with the D.SPECT CZT camera in the forward-leaning biker position, with head-to-head comparisons with more conventional recordings proposed in supine or sitting positions.

\section{MATERIALS AND METHODS}

\section{Study Population}

The study patients were prospectively selected if, first, they had been routinely referred to our department for a single-day stress-first 
${ }^{99 m}$ Tc-sestamibi SPECT protocol performed with a high-sensitivity CZT camera (D.SPECT) $(15,16)$; second, resting SPECT was subsequently indicated on the basis of the initial stress SPECT images (15); third, they were in sinus rhythm, without significant arrhythmia and left bundle branch block; and fourth, they agreed to participate and to sign the informed consent form.

As a part of the study protocol, the routine resting SPECT recorded in the biker position was immediately followed by an additional resting SPECT recording either in a supine position (if they were investigated during a week with a paired number) or in a back-leaning sitting position (if they were investigated during a week with an unpaired number).

The protocol was approved by an Ethics Committee (CPP agreement 16.04.01) and released on the ClinicalTrials.gov site under the identifier NCT02872545.

\section{Stress Tests and Tracer Injection}

The stress tests were performed as described previously $(15,16)$, with exercise performed in the upright position on a bicycle ergometer or with a pharmacologic test conducted with dipyridamole $(0.56$ $\left.\mathrm{mg} \cdot \mathrm{kg}^{-1}\right)$ or regadenoson $(400 \mu \mathrm{g})$.

The ${ }^{99} \mathrm{~m}$ Tc-sestamibi activities injected at stress ranged between 80 and $260 \mathrm{MBq}$ according to body weight as previously described in detail elsewhere (15). The ${ }^{99 \mathrm{~m}} \mathrm{Tc}$-sestamibi activities injected at rest were 3 -fold higher than those injected at stress, although no rest injection was routinely scheduled when the findings of the stress SPECT and gated SPECT images were deemed definitely normal (15).

\section{Acquisition and Reconstruction of D.SPECT Images}

The electrocardiography-triggered SPECT acquisitions were initiated approximately 30-60 min after tracer injection, with the patient being seated in a forward-leaning position, resembling that of a motorbike rider (15). As detailed in Supplemental Figure 1 (supplemental materials are available at http://jnm.snmjournals.org), the patients were set astride the armchair of the camera, with the chest wall leaning on the camera head (commonly orientated at $35^{\circ}$ from vertical) and the patient's back being set with the arms placed orthogonal to the thorax.

As already detailed elsewhere $(15,16)$, each projection was set to target the recording of approximately 500 myocardial kilocounts, leading to recording times of no more than $10 \mathrm{~min}$ for stress acquisitions and almost $4 \mathrm{~min}$ for rest acquisitions.

The routine resting SPECT recording in the biker position was immediately followed by an additional resting SPECT recording with the same parameters, except that patients were either in a strictly supine position with the left arm placed away from the field of view (Fig. 1) or in a back-leaning sitting position with the back rearward at $30^{\circ}$ from vertical, as currently proposed for this camera (Fig. 1) (17).

Image reconstruction was conducted using the parameters already described elsewhere for SPECT (15) and gated SPECT (16).

\section{Analysis of D.SPECT Images}

The D.SPECT data were collected by a single experienced observer, and the results from a second observer were used to assess reproducibility. These analyses involved a combined assessment of the stress and rest SPECT images presented in random order and a masked manner, without prior knowledge of any other medical data or of the position in which the resting SPECT was recorded. In total, 160 combined assessments of stress and rest SPECT images were considered in these analyses since each of the 80 patients had 2 sets of rest SPECT images.

The segmental left ventricular (LV) analysis was performed with a 17-segment model (Fig. 2), with exclusion of the 2 proximal septal segments, corresponding to the outflow tract and membranous septum,

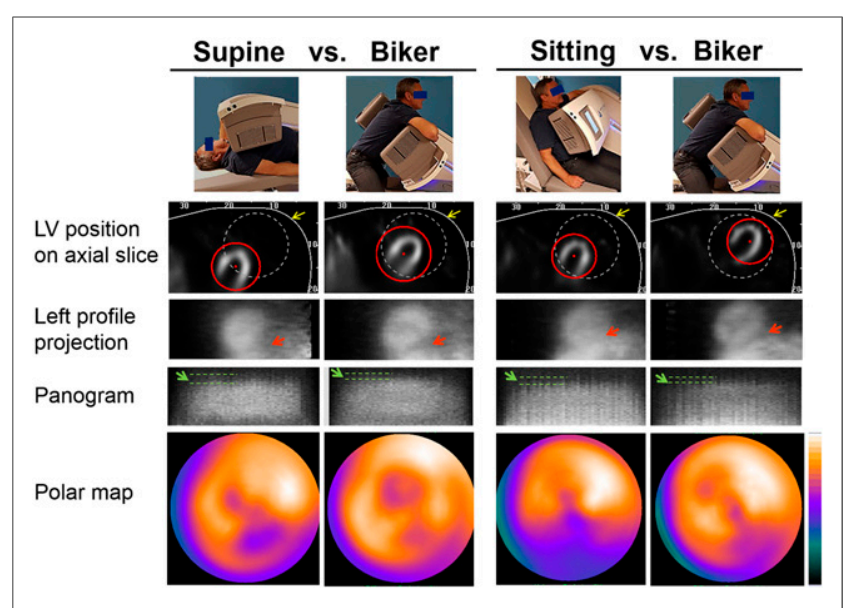

FIGURE 1. Schematic representation of patient positions (top) and of SPECT data from 2 patients belonging to supine and sitting subgroups, respectively, and who were ultimately considered as having attenuation artifacts (bottom). Biker position provides shorter distance between LV and camera head (yellow arrows) on axial tomographic slices, better separation of inferior wall from subphrenic organs (red arrows) on left profile projections, lower amplitude of longitudinal motions of upper LV border on panograms (green arrows, comparing supine with biker positions), and less severe inferior attenuation artifacts on polar maps.

and with the image display provided from the Quantitative Gated SPECT and Quantitative Perfusion SPECT software (Cedars-Sinai Medical Center) $(15,16)$. As previously detailed, myocardial perfusion was assessed visually using a 4-point grading system, and only segments with a moderate to severe reduction at stress were considered to correspond to an abnormal stress uptake (15). Of these, segments with reversible defects, thus corresponding to a pattern of ischemia, were defined as those with an increase in uptake score on the rest acquisition (15). The other stress-defect segments were considered to have a fixed defect, likely corresponding to a pattern of myocardial infarction when the segment's contractility was also found abnormal at gated SPECT (15).

Segmental contractility was assessed through a combined analysis of wall motion and of the systolic increase in myocardial count, a method that compares well with results from cardiac MRI, as previously shown with the same camera (16).

Segments with a high probability of attenuation artifacts were additionally defined on the resting SPECT acquisitions as those showing a decrease in the ${ }^{99 \mathrm{~m}} \mathrm{Tc}$-sestamibi uptake, defined as a mean uptake of less than $65 \%$ of the maximal LV voxel value, a threshold currently used to identify areas with myocardial infarction on rest SPECT $(18,19)$; no pattern of ischemia (i.e., no reversibility from stress defects); and a strictly normal contractility at gated SPECT (i.e., with motion and thickening both deemed definitely normal (16)).

Three additional parameters were extracted from each resting SPECT recording: a tomographic count sensitivity in the LV area, expressed in recorded counts per minute and per megabecquerel of injected activity $(20,21)$; a heart-to-detector distance, which was computed as the distance between the middle part of the camera head (i.e., the angle setting between the 2 half-parts) and the coordinate of the center of the elliptic area placed around the LV before SPECT recording [Fig. 1]); and the amplitude of the longitudinal cardiac motion, which was assessed according to the mean amplitude of the changes in the upper limit of the LV signal on panograms (22). This last parameter consists of quality control images provided routinely by the D.SPECT for detecting longitudinal heart displacement. More precisely, the counts from all detectors are summed and represented 


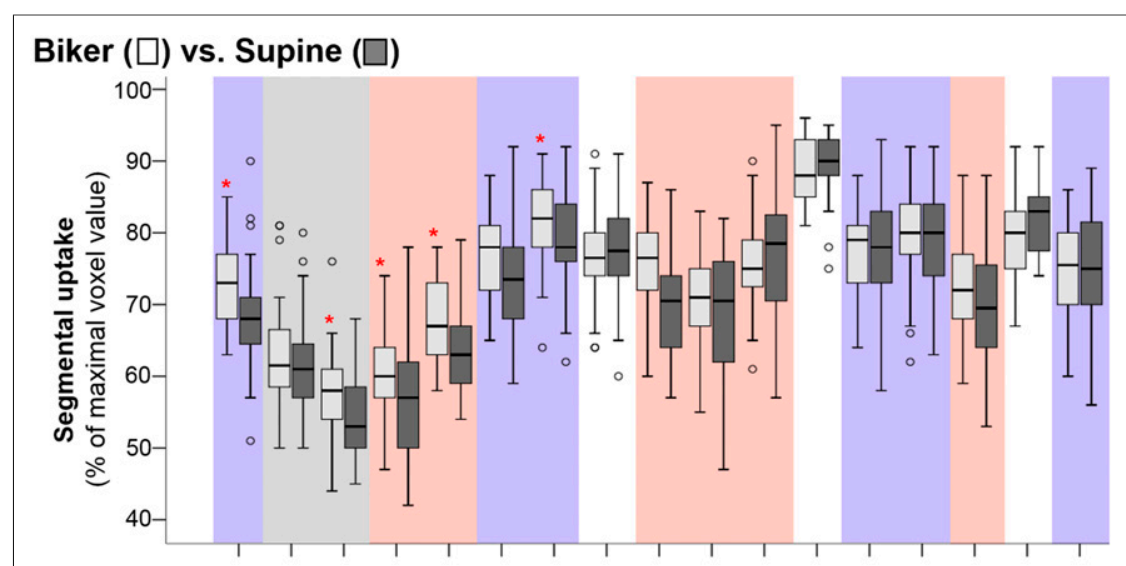

Biker ( $\square$ ) vs. Sitting ( $\square$ )

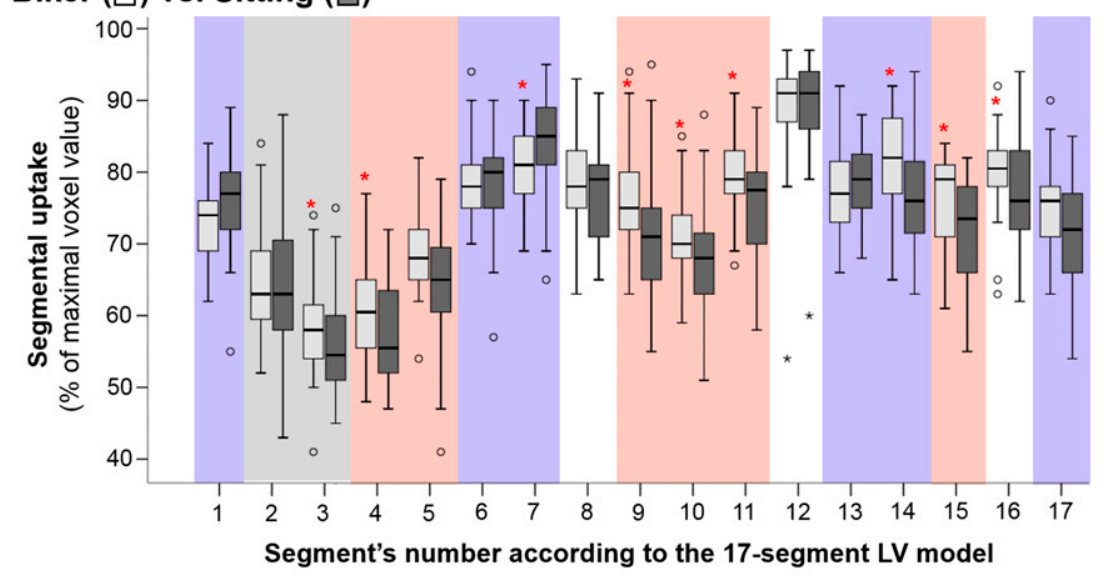

\section{7-segment LV model}

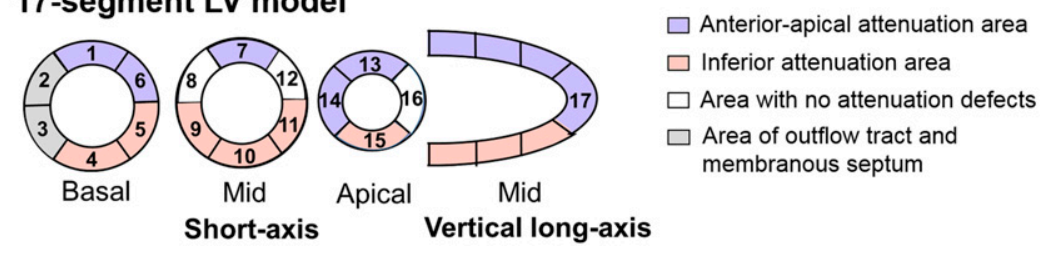

FIGURE 2. Per-segment distribution of uptake values recorded with biker position, as compared with those obtained in same patients in either supine (top) or sitting (bottom) positions, after exclusion of segments with necrotic or ischemic pattern (with $n$ ranging from 32 to 40 in each segment's group). Also shown is schematic representation of 17-segment LV model and of inferior and anterior-apical areas where attenuated segments were identified. Segments 2 and 3 , corresponding to outflow tract and membranous septum, were excluded from these areas. ${ }^{*} P<$ 0.05 for paired comparisons between patient positions.

through contiguous vertical lines for each of the 120 consecutive projection positions, each projection lasting approximately $2 \mathrm{~s}$ during resting SPECT [Fig. 1]). This mean amplitude of longitudinal motions was scored visually with the help of a graded scale and a 4-point grading system $(1,<5 \mathrm{~mm} ; 2, \geq 5$ and $<10 \mathrm{~mm} ; 3, \geq 10$ and $<15 \mathrm{~mm}$; and $4, \geq 15 \mathrm{~mm}$ ).

\section{Statistical Analysis}

Continuous variables are reported as mean $\pm \mathrm{SD}$, and categoric variables as percentages. The interobserver reproducibility of segment classification was analyzed through concordance with $\kappa$-values. Unpaired and paired comparisons between continuous variables were performed with the Mann-Whitney and Wilcoxon rank sum tests, respectively, whereas unpaired comparisons between categoric variables were performed with $\chi^{2}$ tests. For all tests, a $P$ value of less than 0.05 was considered to reflect a significant difference.

\section{RESULTS}

Patient characteristics are summarized in Table 1 for the overall study population, as well as for the supine and sitting subgroups. Mean age was $62 \pm 10 \mathrm{y} ; 24(30 \%)$ were obese (as defined by a body mass index $>$ $30 \mathrm{~kg} \cdot \mathrm{m}^{-2}$ ); and $15(19 \%)$ were women, of whom only $5(6 \%)$ were obese. The sitting subgroup was of moderately older age and with a lower proportion of women than the supine subgroup (Table 1).

Reproducibility between the 2 observers was close to excellent, with a global $\kappa$-score of 0.74 for identifying the segments showing a pattern of attenuation artifact. This reproducibility was good to very good for the identification of segments showing a pattern of ischemia or of infarction, with respective к-scores of 0.55 and 0.71 .

Among the total 2,400 analyzed segments (15 segments for each of the 160 sets of stress and rest images), 205 (8.5\%) had a pattern of attenuation artifact. Up to $86 \%$ of these attenuated segments were in a large inferior area, whereas the remaining $14 \%$ were in an anterior and apical area. These 2 attenuation areas are displayed on the schematic representation of the 17-segment model in Figure 2.

As detailed in Table 2, there were no significant differences between the SPECT results obtained with the biker position and with the sitting or supine position with regard to the mean number of segments identified as having a pattern of ischemia or infarction. By contrast, the mean number of segments showing a pattern of attenuation artifact was markedly lower with the biker position than with the supine position $(1.10 \pm 1.01$ vs. $1.90 \pm 1.74, P=$ $0.010)$ or the sitting position $(0.75 \pm 0.93$ vs. $1.38 \pm 1.60, P=0.011)$. These differences remained significant when the analyses were restricted to the inferior attenuation area defined above but not when restricted to the anterior and apical attenuation area, although the comparison between biker and supine positions was at the borderline of significance for the latter $(P=0.08)$ (Table 2).

A mean uptake value was computed for each LV segment, after having excluded all those associated with a pattern of ischemia or infarction on analyses obtained with either the biker, supine, or sitting rest SPECT images. The uptake values of these normally perfused segments are displayed through plot boxes in Figure 2, yielding evidence of higher uptakes with the biker than with the sitting or supine positions for several segments, particularly those located in the proximal part of the inferior attenuation area.

As depicted in Table 2, the biker position was additionally associated with a lower heart-to-camera-head distance than the 
TABLE 1

Main Characteristics of Overall Study Population and of 2 Subgroups in Which Additional Rest SPECT Was Recorded in Sitting or Supine Position

\begin{tabular}{|lccc}
\hline \multicolumn{1}{c}{ Characteristic } & Overall study population $(n=80)$ & Sitting subgroup $(n=40)$ & Supine subgroup $(n=40)$ \\
\hline Age $(\mathrm{y})$ & $62 \pm 10$ & $64 \pm 12$ & $59 \pm 8^{*}$ \\
\hline Female sex & $15(19)$ & $4(10)$ & $11(27)^{\star}$ \\
\hline Body mass index $\left(\mathrm{kg} \cdot \mathrm{m}^{-2}\right)$ & $29 \pm 5$ & $28 \pm 5$ & $29 \pm 5$ \\
\hline $30 \mathrm{~kg} \cdot \mathrm{m}^{-2}$ & $24(30)$ & $12(30)$ & $12(30)$ \\
\hline History of myocardial infarction & $27(34)$ & $17(42)$ & $10(25)$ \\
\hline History of coronary artery bypass & $9(11)$ & $6(15)$ & $3(7)$ \\
\hline History of coronary angioplasty & $33(41)$ & $21(52)$ & $12(30)$ \\
\hline Recent history of chest pain & $32(40)$ & $17(42)$ & $15(38)$ \\
\hline Exercise stress test & $70(87)$ & $33(82)$ & $37(92)$ \\
\hline Pharmacologic stress test & $10(12)$ & $7(17)$ & $3(7)$ \\
\hline Segments with stress defects & $2.5 \pm 2.7$ & $3.0 \pm 2.9$ & $2.0 \pm 2.4$ \\
\hline
\end{tabular}

${ }^{\star} P<0.05$ for paired comparisons between sitting and supine subgroups.

Qualitative data are expressed as numbers, followed by percentages in parentheses; continuous data are expressed as mean \pm SD.

supine and sitting positions (both $P<0.001$ ), as well as with a lower count sensitivity and lower LV longitudinal motion amplitude than the supine position $(P \leq 0.001)$.

These results are illustrated in Figure 1 by representative examples of SPECT images obtained in 2 patients for whom the additional rest SPECT was recorded in sitting or supine position, as well as in Supplemental Figure 2, where SPECT images from an overall recording protocol are displayed (biker stress, biker rest, and supine rest SPECT).

\section{DISCUSSION}

Despite their improved performance characteristics, cardiac CZT cameras are still considered to provide suboptimal specificity for identifying significant coronary artery disease (23), and attenuation artifacts potentially play a substantial role in this setting. Indeed, attenuation artifacts are at least as severe with Anger cameras as with CZT cameras (1) and, to date, are far from being fully prevented or detected by the various methodologies and strategies elaborated for this purpose (2-15).

In the present prospective randomized study conducted with the D.SPECT CZT camera, attenuation artifacts were shown to be strongly reduced in patients positioned in a semireclining bikerlike position comparatively to the more conventional sitting and supine positions. This biker position was initially developed as an equivalent to the prone position adapted to the particular geometry of the D.SPECT camera, for which image recording in the prone position may not be proposed. This position is seemingly more comfortable than the prone position, with the patient's shoulders being less stretched back and breathing less affected by lung

TABLE 2

Paired Comparisons Between SPECT Data Recorded in Biker Position and in Either Sitting $(n=40)$ or Supine $(n=40)$ Position

\begin{tabular}{|c|c|c|c|c|c|c|}
\hline Parameter & Biker & Sitting & $P$ & Biker & Supine & $P$ \\
\hline Longitudinal motion score & $1.3 \pm 0.6$ & $1.3 \pm 0.9$ & NS & $1.6 \pm 0.8$ & $2.5 \pm 1.6$ & $<0.001$ \\
\hline Heart-to-camera-head distance $(\mathrm{cm})$ & $18.3 \pm 2.3$ & $20.1 \pm 2.4$ & $<0.001$ & $18.3 \pm 1.6$ & $20.2 \pm 2.5$ & $<0.001$ \\
\hline Count sensitivity (counts/min/MBq) & $586 \pm 170$ & $600 \pm 173$ & NS & $557 \pm 152$ & $609 \pm 171$ & 0.001 \\
\hline \multicolumn{7}{|l|}{ Defect extent (mean number of segments) } \\
\hline Ischemic pattern & $1.58 \pm 2.31$ & $1.35 \pm 2.23$ & NS & $1.0 \pm 1.7$ & $1.2 \pm 1.5$ & NS \\
\hline Infarction pattern & $1.38 \pm 2.62$ & $1.40 \pm 2.28$ & NS & $0.9 \pm 2.0$ & $0.6 \pm 1.3$ & NS \\
\hline Attenuation artifact pattern & $0.75 \pm 0.93$ & $1.38 \pm 1.60$ & 0.011 & $1.10 \pm 1.00$ & $1.90 \pm 1.74$ & 0.010 \\
\hline Inferior attenuation area* & $0.70 \pm 0.91$ & $1.28 \pm 1.43$ & 0.006 & $0.93 \pm 0.94$ & $1.52 \pm 1.58$ & 0.046 \\
\hline Anterior and apical attenuation area ${ }^{\dagger}$ & $0.05 \pm 0.22$ & $0.10 \pm 0.30$ & NS & $0.18 \pm 0.38$ & $0.38 \pm 0.70$ & 0.083 \\
\hline
\end{tabular}

${ }^{*}$ Corresponding to segments belonging to inferior attenuation area described at bottom of Figure 2.

${ }^{\dagger}$ Corresponding to segments belonging to anterior and apical attenuation area described at bottom of Figure 2.

$\mathrm{NS}=$ nonsignificant with $P>0.10$. Data are mean \pm SD. 
compression due to thoracic weight. In our experience, the biker position is well tolerated, even in patients with severe LV dysfunction. No more than $5 \%$ of the patients referred to our department for myocardial perfusion SPECT are unable to complete this examination in the biker position, mostly because of extreme physical exhaustion or hip problems hampering their sitting astride the camera armchair.

As detailed in Table 2 and Figure 2, this biker position was shown here to reduce myocardial attenuation and corresponding artifacts, as compared with the sitting or supine position, with this reduction mainly involving the basal portion of the inferior area, similarly to that previously reported for the prone versus the supine position.

As observed on the LV projection profiles in Figure 1, this reduction in inferior attenuation was moreover associated with easier delineation and separation of the inferior LV wall from the attenuating subphrenic organs. This observation can be partly explained by an upward LV displacement, as previously documented for prone comparatively to supine positions (24). The biker position was additionally associated with a trend toward a lower rate of anteroseptal and apical defects, as compared with the supine position, together with a higher normal segmental uptake in the proximal and median portion of the anterior wall (segments 1 and 7 in Fig. 2). This observation is in contrast to higher attenuation rates documented for the prone position in the anterior area $(11,13)$. The normal segmental uptake of the same proximal and median portion of the anterior wall was found even higher with the sitting position (Fig. 2), strengthening the general consideration that the different patient positions lead to specific attenuation patterns and are thus not interchangeable for further patient monitoring.

The biker position was associated with 2 additional properties that could further favor image quality. The first is the small amplitude of longitudinal cardiac motion. This property, which was also observed for the sitting position here, may likely be explained by lower amplitudes of breathing-related diaphragmatic motion, as already documented for upright versus supine positions in previous MRI and ultrasound studies $(25,26)$. According to the scores obtained from panograms (Fig. 1), this longitudinal LV motion did not exceed $5 \mathrm{~mm}$ on average for both upright sitting and biker positions, whereas it ranged between 5 and $10 \mathrm{~mm}$ for the supine position. Although the true clinical impact of this finding remains to be properly defined, it may be hypothesized to play a role additional to that of the upward LV shift, in the enhanced separation documented with the biker position between the inferior wall and subphrenic organs.

A second additional property of the biker position, also likely to enhance spatial resolution, is a lower heart-to-detector distance (18 $\mathrm{cm}$, on average), when compared with the 2 other patient positions $(20 \mathrm{~cm}$, on average). Indeed, in the biker position, the anterior chest wall directly leans on the surface of the camera head. By contrast, the camera head needs to be placed a few centimeters from the anterior chest wall in the sitting and supine positions.

An almost $10 \%$ lower tomographic count sensitivity was documented in the present study for the biker position than for the supine position. This decrease is likely explained by the short heart-to-detector distance, leading to positioning of the $\mathrm{LV}$ in an area where count sensitivity is slightly lower than in a more remote area, a property that was confirmed by further phantom experiments (results not shown).
A limitation of the study is that the presence of attenuation artifacts could not be confirmed by the absence of any coronary stenosis at angiography. Only a few patients had subsequent coronary angiography, especially among those exhibiting mainly attenuation artifacts. However, in a previous report, the sensitivity for identifying patients with coronary stenosis was found to be rather high $(88 \%)$ for stress SPECT recorded with the biker position (15), and the rate of normal stress D.SPECT images reached $97 \%$ in patients with a less than $10 \%$ likelihood of coronary artery disease (normalcy rate), strengthening the general consideration of a rather low rate of artifacts with the biker position (15).

It should additionally be kept in mind that only the resting SPECT was repeated here for further comparisons of patient positions and that only patients with abnormal or at least doubtful stress SPECT findings in our current biker position could be included. Thereby, it is likely that this study as designed does not allow precise determination of the actual rates of attenuation artifacts, or of diagnostic confidence and accuracy, in the different patient positions.

It must also be recognized that most attenuation artifacts could be easily identified through an expert visual analysis. However, minimizing these artifacts and improving the uptake of attenuated segments is likely suitable for better assessing myocardial ischemia and necrosis.

\section{CONCLUSION}

Myocardial perfusion images from the D.SPECT camera are enhanced for patients positioned in a forward-leaning biker position rather than in the more conventional sitting or supine position. This enhancement involves not only a lower rate of attenuation artifacts but also lower heart-to-detector distances and lower amplitudes in breathing-related cardiac motion. These properties are likely to favor image quality and, potentially, diagnostic accuracy, although this latter feature remains to be further assessed in dedicated ancillary studies.

\section{DISCLOSURE}

No potential conflict of interest relevant to this article was reported.

\section{REFERENCES}

1. Oddstig J, Martinsson E, Jögi J, Engblom H, Hindorf C. Differences in attenuation pattern in myocardial SPECT between CZT and conventional gamma cameras. J Nucl Cardiol. May 23, 2018 [Epub ahead of print].

2. Nakaya K, Onoguchi M, Nishimura Y, et al. Comparison between prone and upright imaging of the inferior wall using ${ }^{201} \mathrm{TlCl}$ myocardial perfusion SPECT. J Nucl Med Technol. 2017;45:304-308.

3. Caobelli F, Akin M, Thackeray JT, et al. Diagnostic accuracy of cadmium-zinctelluride-based myocardial perfusion SPECT: impact of attenuation correction using a co-registered external computed tomography. Eur Heart J Cardiovasc Imaging. 2016;17:1036-1043.

4. Tout D, Tonge C, Austin P, Adams J, Arumugam P. A comparison between upright and supine myocardial perfusion imaging with attenuation correction. Nucl Med Commun. 2014;35:832-838.

5. Ito S, Endo A, Okada T, et al. Comparison of CTAC and prone imaging for the detection of coronary artery disease using CZT SPECT. Ann Nucl Med. 2017; 31:629-635

6. Taillefer R, DePuey EG, Udelson JE, Beller GA, Latour Y, Reeves F. Comparative diagnostic accuracy of Tl-201 and Tc-99m sestamibi SPECT imaging (perfusion and ECG-gated SPECT) in detecting coronary artery disease in women. J Am Coll Cardiol. 1997;29:69-77. 
7. Genovesi D, Giorgetti A, Gimelli A, et al. Impact of attenuation correction and gated acquisition in SPECT myocardial perfusion imaging: results of the multicentre SPAG (SPECT attenuation correction vs gated) study. Eur J Nucl Med Mol Imaging. 2011;38:1890-1898.

8. Kennedy JA, Israel O, Frenkel A. Directions and magnitudes of misregistration of CT attenuation-corrected myocardial perfusion studies: incidence, impact on image quality, and guidance for reregistration. J Nucl Med. 2009;50:1471-1478.

9. Nakaya K, Onoguchi M, Nishimura Y, et al. Criteria for the addition of prone imaging to myocardial perfusion single-photon emission computed tomography for inferior wall. Nucl Med Commun. 2017;38:748-755.

10. Ceylan Gunay E, Erdogan A, Yalcin H, Ozcan Kara P. Prone imaging allows efficient radiopharmaceutical usage by obviating the necessity of a rest study in Tc-99m-methoxyisobutylisonitrile myocardial perfusion scintigraphy. Nucl Med Commun. 2011;32:284-288.

11. Nishiyama Y, Miyagawa M, Kawaguchi N, et al. Combined supine and prone myocardial perfusion single-photon emission computed tomography with a cadmium zinc telluride camera for detection of coronary artery disease. Circ J. 2014;78:1169-1175.

12. Peterson PN, Parker JA, Tepper MR, Hauser TH, English J, Danias PG. Prone SPECT myocardial perfusion imaging is associated with less cardiac drift during the acquisition duration than imaging in the supine position. Nucl Med Commun. 2005;26:115-117.

13. Kiat H, Van Train KF, Friedman JD, et al. Quantitative stress-redistribution thallium-201 SPECT using prone imaging: methodologic development and validation. J Nucl Med. 1992;33:1509-1515.

14. Nakazato R, Tamarappoo BK, Kang X, et al. Quantitative upright-supine highspeed SPECT myocardial perfusion imaging for detection of coronary artery disease: correlation with invasive coronary angiography. J Nucl Med. 2010;51: 1724-1731.

15. Perrin M, Djaballah W, Moulin F, et al. Stress-first protocol for myocardial perfusion SPECT imaging with semiconductor cameras: high diagnostic performances with significant reduction in patient radiation doses. Eur J Nucl Med Mol Imaging. 2015;42:1004-1011.

16. Claudin M, Imbert L, Djaballah W, et al. Routine evaluation of left ventricular function using CZT-SPECT, with low injected activities and limited recording times. J Nucl Cardiol. 2018;25:249-256.
17. Jameria ZA, Abdallah M, Dwivedi A, et al. Computer derived transient ischemic dilation ratio for identifying extensive coronary artery disease using a CZT camera and imaging in the upright position. J Nucl Cardiol. 2017;24: 1702-1708.

18. De Geeter F, Franken PR, Knapp FF Jr, Bossuyt A. Relationship between blood flow and fatty acid metabolism in subacute myocardial infarction: a study by means of ${ }^{99 \mathrm{~m} T c-s e s t a m i b i}$ and ${ }^{123}$ I-beta-methyl-iodo-phenyl pentadecanoic acid. Eur J Nucl Med. 1994;21:283-291.

19. Sciagrà R, Pellegri M, Pupi A, et al. Prognostic implications of Tc-99m sestamibi viability imaging and subsequent therapeutic strategy in patients with chronic coronary artery disease and left ventricular dysfunction. J Am Coll Cardiol. 2000;36:739-745.

20. Imbert L, Poussier S, Franken PR, et al. Compared performance of high-sensitivity cameras dedicated to myocardial perfusion SPECT: a comprehensive analysis of phantom and human images. J Nucl Med. 2012;53:1897-1903.

21. Verger A, Imbert L, Yagdigul Y, et al. Factors affecting the myocardial activity acquired during exercise SPECT with a high-sensitivity cardiac CZT camera as compared with conventional Anger camera. Eur J Nucl Med Mol Imaging. 2014;41:522-528.

22. Allie R, Hutton BF, Prvulovich E, Bomanji J, Michopoulou S, Ben-Haim S. Pitfalls and artifacts using the D-SPECT dedicated cardiac camera. J Nucl Cardiol. 2016;23:301-310.

23. Nudi F, Iskandrian AE, Schillaci O, Peruzzi M, Frati G, Biondi-Zoccai G. Diagnostic accuracy of myocardial perfusion imaging with CZT technology: systemic review and meta-analysis of comparison with invasive coronary angiography. JACC Cardiovasc Imaging. 2017;10:787-794.

24. Esquerré JP, Coca FJ, Martinez SJ, Guiraud RF. Prone decubitus: a solution to inferior wall attenuation in thallium-201 myocardial tomography. J Nucl Med. 1989;30:398-401.

25. Houston JG, Morris AD, Howie CA, Reid JL, McMillan N. Technical report: quantitative assessment of diaphragmatic movement-a reproducible method using ultrasound. Clin Radiol. 1992;46:405-407.

26. Takazakura R, Takahashi M, Nitta N, Murata K. Diaphragmatic motion in the sitting and supine positions: healthy subject study using a vertically open magnetic resonance system. J Magn Reson Imaging. 2004;19:605-609. 